\title{
A comparison of 23-gauge and 20-gauge vitrectomy for proliferative sickle cell retinopathy - clinical outcomes and surgical management
}

\author{
Jason $\mathrm{Ho}^{1} \cdot$ Anna Grabowska' $\cdot$ Marta Ugarte ${ }^{2,3} \cdot$ Mahiul MK Muqit $^{1,3}$ \\ Received: 2 February 2018 / Revised: 23 March 2018 / Accepted: 25 April 2018 / Published online: 22 May 2018 \\ (c) The Royal College of Ophthalmologists 2018
}

\begin{abstract}
Aims To report anatomical and functional outcomes in patients with proliferative sickle retinopathy (PSR) who underwent 23 -gauge (23G) and 20-gauge (20G) vitrectomy.

Methods Retrospective consecutive case series of patients who underwent vitreoretinal intervention for complications of PSR between April 2009 and February 2015. Operations were performed at a tertiary referral centre, Moorfields Eye Hospital. Visual acuity and anatomical success rates were evaluated for PSR complicated by retinal detachment, tractional vitreous haemorrhage and macular hole. Proliferative diabetic retinopathy cases were excluded.

Results A total of 71 eyes (63 patients) underwent vitreoretinal surgery for PSR complications with 26 months mean followup. Primary indications were: tractional retinal detachment (TRD, $n=17)$, TRD with rhegmatogenous retinal detachment $(n$ $=16$ ), rhegmatogenous retinal detachment $(n=5$, macula-on: 1 , macula-off: 4$)$, vitreous haemorrhage $(n=19)$, epiretinal membrane $(n=6)$, and full thickness macula hole $(n=8)$. Thirty-nine cases underwent 20G vitrectomy, and 23G surgery was performed in 32 eyes. Mean best corrected visual acuity (BCVA) improved from pre-operative 1.30 LogMAR to final BCVA of 0.74 LogMAR $(p<0.01$, paired $t$-test). 23G vitrectomy yielded slightly better 32 vs. 25 ETDRS-letter improvement compared with $20 \mathrm{G}$ vitrectomy ( $p=0.60$, NS, unpaired $t$-test). $23 \mathrm{G}$ was associated with fewer per-operative complications (23G, $18 \%$ vs. $20 \mathrm{G}, 13 \%$ ). The subset of 38 eyes with retinal detachment demonstrated $79 \%$ primary reattachment rate, and a smaller BCVA improvement of 3 lines $(p=0.07$, paired $t$-test).

Conclusions Surgical intervention for complicated PSR can preserve and/or improve vision although the degree of visual acuity stabilisation remains guarded in tractional/rhegmatogenous detachments. $23 \mathrm{G}$ vitrectomy may give a better functional outcome with lower per-operative complication rates.
\end{abstract}

\section{Introduction}

Sickle cell is one of the most common severe monogenic disorders worldwide with autosomal recessive inheritance [1].

Electronic supplementary material The online version of this article (https://doi.org/10.1038/s41433-018-0127-y) contains supplementary material, which is available to authorized users.

Mahiul MK Muqit

Mahi.Muqit@Moorfields.nhs.uk

1 Vitreoretinal Service, Moorfields Eye Hospital, 162 City Rd, London EC1V 2PD, UK

2 Medical Retina Service, Moorfields Eye Hospital, 162 City Rd, London EC1V 2PD, UK

3 Institute of Ophthalmology, University College London, 11-43 Bath St, London EC1V 9EL, UK
Sight-threatening complications in sickle cell disease (SCD) are mainly due to proliferative sickle retinopathy (PSR), which is secondary to occlusion of the peripheral retinal vasculature that in turn leads to retinal ischaemia and proliferation of new blood vessels with characteristic sea-fan appearance. The incidence of PSR is higher in HbSC disease, increases with age and is relatively common between 15 and 29 years of age [2]. Visual loss in PSR is commonly due to vitreous haemorrhage $(\mathrm{VH})$, macula-threatening tractional and/or rhegmatogenous retinal detachment (TRD/RRD), epiretinal membrane (ERM) and macular hole (MH) [3]. Despite the high prevalence of autoinfarction above $30 \%$, permanent visual loss occurs in $10-12 \%$ of patients with PSR [3, 4]. Only a limited number of small studies demonstrate improved outcomes from surgery for complications of PSR [5-7].

We conducted a case series study to compare the outcomes from 23-gauge (23G) and 20-gauge vitrectomy 
(20G) for PSR and to explore contemporary anatomical and visual outcomes. We also qualitatively analysed the main patient subgroups of interest within our cohort, namely patients with retinal detachments, macula holes and prior PRP laser treatment.

\section{Materials and methods}

This was a retrospective consecutive case series of all patients undergoing vitreoretinal surgery from April 2009 to February 2015 for PSR at the Moorfields Eye Hospital. This large tertiary eye hospital serves a diverse population with a significant Afro-Caribbean ethnic population in the London area. All operations were performed by subspecialist vitreoretinal consultants or senior fellows. The data were collected from electronic patient records and validated with original clinical notes. They included demographics, sickle cell status, best corrected visual acuity (BCVA), lens status, operative procedures, final anatomical post-operative outcome at last follow-up, further (secondary) procedures and duration of follow-up. Indications for surgical management included recurrent or non-clearing $\mathrm{VH}, \mathrm{ERM}, \mathrm{TRD}$ and combined TRD with RRD. Standard 20 or $23 \mathrm{G}$ pars plana vitrectomy (PPV) was performed using a BIOM wide-angle viewing system under subtenon's local anaesthesia or general anaesthesia. No exchange transfusions or scleral buckling procedures were performed. The operating technique was surgeon-dependent, and consultant-supervised for fellow-led surgery. Cases with associated proliferative diabetic retinopathy were specifically excluded from this study.

The primary outcome was defined as change in BCVA until final follow-up. Secondary outcome measures included primary anatomical retinal reattachment rate without tamponade, macular hole closure and post-operative complications. The study adhered to the tenets of the Declaration of Helsinki and was approved by the Moorfields Eye Hospital Audit Committee.

\section{Statistical analysis}

Snellen BCVA was reviewed and converted into LogMAR acuity for statistical analysis, which was performed on the full dataset. GraphPad Prism v6 software was used for both descriptive and numerical statistical comparisons. Paired $t$ tests were performed and a $p$-value $<0.05$ was considered statistically significant. The figures depict standard error of the mean bars for data value.

\section{Results}

Seventy-one eyes from 63 patients with PSR met the study criteria during this 6-year study period. There were 34 male and 29 female patients with a mean age of 41.7 years (range 16-71). Sixty-eight (96\%) cases were phakic prior to primary retinal surgery, and 61 out of $71(86 \%)$ of operations were undertaken with local anaesthesia. Thirty-nine cases underwent 20G PPV and 32 cases underwent 23G PPV. Three primary lensectomies and one phacoemulsification with intraocular lens implantation were performed. The mean duration of symptoms prior to surgery was 34 weeks (range 1-364 weeks) and mean post-operative follow-up period was 26 months. The distribution of haemoglobin subtypes in our population and indications for surgery are also shown in Table 1.

The mean BCVA improved from 1.30 LogMAR preoperatively to 0.74 LogMAR at final follow-up $(p<0.01$, paired $t$-test) as depicted in Fig. 1. This change is equivalent to 5.5 ETDRS lines. Overall, $49 \%$ attained a final postoperative BCVA of $0.3 \operatorname{LogMAR}$ or better. In $83 \%$, the final post-operative BCVA was equal to or better compared to baseline.

\section{Surgery: 23G vs. $20 G$}

Figure $1 \mathrm{~b} 23 \mathrm{G}$ vitrectomy surgery yielded a better overall 0.62 LogMAR improvement in BCVA (32 ETDRS letters) compared to the 0.50 LogMAR gain (25 ETDRS letters) recorded with $20 \mathrm{G}$ instrumentation $(p=0.60, \mathrm{NS}$, unpaired $t$-test). A total of $75.8 \%$ of $23 \mathrm{G}$ cases and $64.1 \%$ of $20 \mathrm{G}$ cases demonstrated an improvement in BCVA following vitrectomy. ( $p=0.30$, Fisher's exact test).

\section{Retinal detachment}

We subanalysed 38 sickle cell retinopathy cases with retinal detachment; five eyes with RRD (macula-on: 1, macula-off: 4); 17 had TRD (macula-on: 4, macula-off: 13); and 16 were combined TRD and RRD (macula-on: 4, macula-off: 12) as shown in Table 1. Regarding intraocular tamponade agents, 20 cases involved primary silicone oil tamponade, which included 7 cases with primary retinectomy.

The final anatomical outcome at last follow-up visit was: $30 / 38(79 \%)$ attached without tamponade, 4/38 (10.5\%) attached under oil, and 4/38 (10.5\%) detached under oil. After primary vitrectomy surgery, 11 cases underwent subsequent cataract surgery and/or removal of oil at a median of 36 weeks (range 10-324 weeks), and 2 cases with attached retina had long-term oil tamponade. In one case, the retina remained detached under oil without further intervention.

In addition, 11 patients underwent a secondary retinal intervention (median number of procedures $=2$ ). In this group, 6 cases were attached, 2 were attached under oil and 3 remained detached under oil.

Following surgery, the mean BCVA for PSR-related retinal detachment cases improved from LogMAR 1.38 to 
LogMAR 1.05 at the final visit $(p=0.07$, paired $t$-test). Post-operative BCVA was maintained/improved in $76 \%$ of vitrectomised PSR eyes, and $23.7 \%$ attained final postoperative BCVA of LogMAR 0.3 or better. A direct comparison between 23 vs. $20 \mathrm{G}$ vitrectomy showed the final overall anatomical reattachment rate was $82 \%$ with $23 \mathrm{G}$ PPV vs. $78 \%$ with $20 \mathrm{G}$ PPV surgery ( $p=0.96$, NS, Fisher's exact test), as shown in Fig. 2.

In Supplemental Fig. 1, a 26-year-old HbSC patient with PSR and a left tractional retinal detachment underwent successful surgical intervention with 23G vitrectomy, delamination, internal limiting membrane (ILM) peel, laser retinopexy and $\mathrm{C} 3 \mathrm{~F} 8$ gas tamponade, with a good surgical outcome and stable retinal re-attachment at 34 months.

\section{Full thickness macular hole (FTMH)}

For 8 cases with primary FTMH, anatomical closure was achieved in 7 of 8 cases with C3F8 gas tamponade $(n=7)$. There was one case of non-closure that used SF6 tamponade, and this hole closed after revisional surgery. Figure $3 \mathrm{a}$ shows the improvement in mean BCVA from $1.053 \mathrm{Log}$ MAR to 0.685 at final follow-up $(p=0.23$, NS).

In Supplemental Fig. 2, a 33-year-old HbSC with PSR and FTMH underwent 23G vitrectomy, delamination, ILM peel and C3F8 gas tamponade surgery. The macular hole closed successfully, and vision improved from LogMAR 1.0 to $\operatorname{LogMAR} 0.6$ at the final post-operative visit, at 20 months.

\section{Scatter PRP}

In total, 14 cases underwent scatter panretinal photocoagulation (PRP). Twelve patients had PRP at the time of surgery, 2 cases underwent pre-operative PRP and 4 had PRP both before and during vitrectomy for sickle retinopathy. For the latter 6 cases, the time interval between PRP and first vitrectomy ranged from 14 months to 5 years. A direct comparison of 6 cases with prior PRP vs. those without prior PRP showed no significant difference in the change in VA after vitrectomy $(p=0.48$, NS, unpaired $t$ test), Fig. 3b.

\section{Complications}

Intra-operatively, six cases of iatrogenic breaks were encountered, 3 in the $23 \mathrm{G}$ group and 3 in the $20 \mathrm{G}$ group. Two giant retinal tears (GRT) occurred in the 20G group. Post-operatively, the $23 \mathrm{G}$ group had one case of fibrinous uveitis, and one case of hypertensive uveitis after $20 \mathrm{G}$ surgery. The uveitis resolved in both cases. One case of post-operative branch retinal artery occlusion was encountered in the $20 \mathrm{G}$ group. There were no recorded instances of 
Fig. 1 a (left) -Mean overall BCVA improvement $(n=71)$ from LogMAR 1.30 preoperatively to LogMAR 0.74 at final follow-up. b (right) Comparison of mean change in BCVA following 23G $(0.62$ LogMAR improvement) vs. 20G vitrectomy surgery $(0.50$ LogMAR gain) for PSR
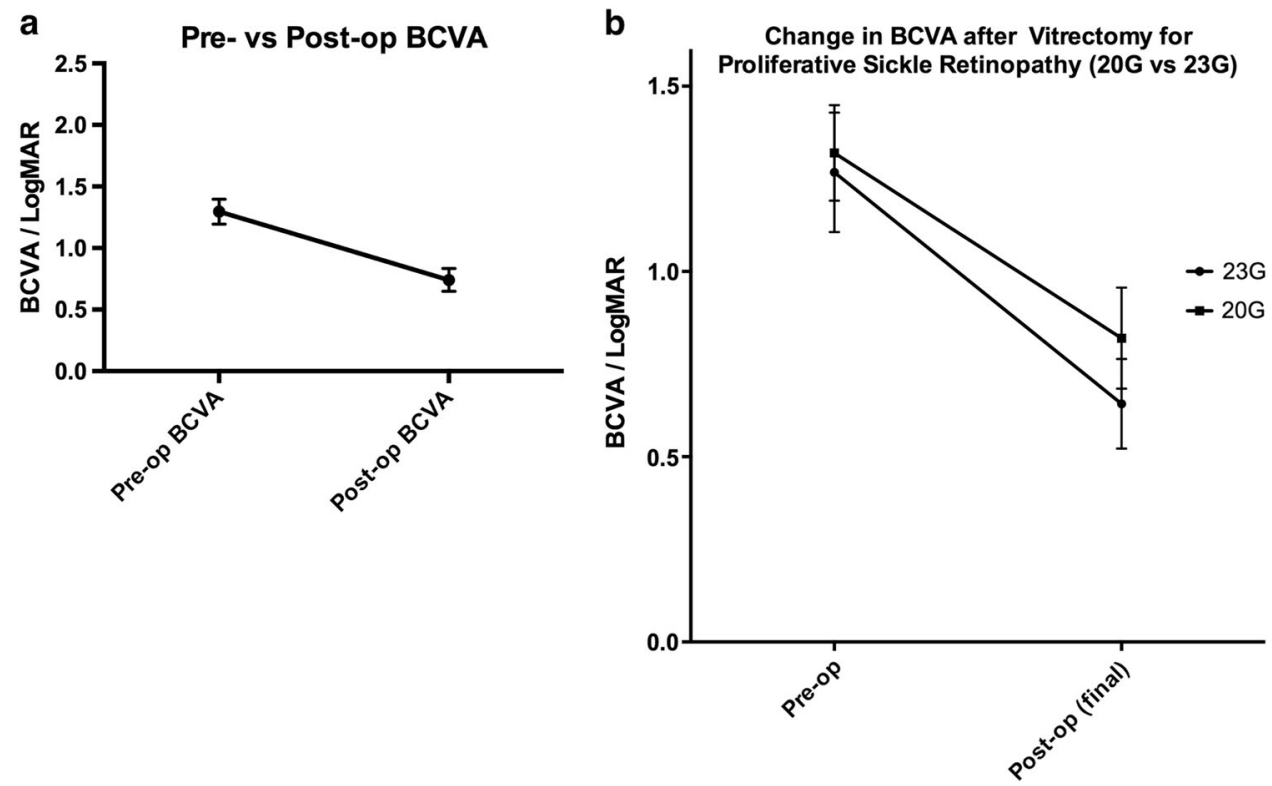

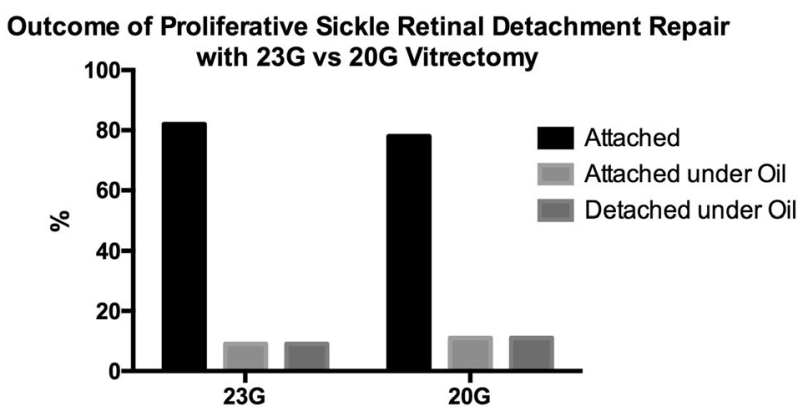

Fig. 2 Final overall anatomical reattachment rate of $82 \%$ with 23G PPV vs. $78 \%$ with $20 \mathrm{G}$ PPV surgery

vitreous cavity haemorrhages or other ischaemic complications during the post-operative follow-up period. This is summarised in Table 2. The mean pre-operative intraocular pressure was $13.6 \mathrm{mmHg}$ which did not rise significantly after surgery to $14.4 \mathrm{mmHg},(p=0.17, \mathrm{NS}$, unpaired $t$-test).

\section{Discussion}

This is the largest study to date that reports favourable visual and anatomical outcomes for the surgical management of patients with complications of PSR. We demonstrate that surgical intervention for sight-threatening macular and retinal complications of PSR can preserve and also improve vision, with a significant final mean gain of 5.5 lines of BCVA validated over a 2-year follow-up period. Compared to the two published studies of surgery for all complications of PSR over the past decade [5, 6], we report a similar proportion of stabilisation/improvement in postoperative BCVA in $83 \%$ of cases.
In agreement with other authors, the degree of stabilisation in visual acuity remains guarded in TRD/RRDs in these patients. However, while the improvement in mean post-operative BCVA did not reach a level of statistical significance, it is encouraging to report that $76 \%$ maintained or improved final BCVA after surgery, which is important when balancing guarded patient expectations whilst offering hope for improvement with surgery in this young patient cohort. The spectrum of vitreoretinal interface disorders that affect patients with PSR reflects the variable outcomes with surgery.

Furthermore, a lower proportion of our patients required additional vitreoretinal interventions (28.9\%) to manage recurrent retinal detachment than the most recent study by Chen et al.[6] (50\%). Surgery in these patients is challenging and other studies have previously reported rates of iatrogenic breaks over $30 \%[5,8]$, which may be attributed to the relatively anterior tractional pathology. Our patients experienced a low-intraoperative complication rate of 12.8 and $9 \%$ for 20 and $23 \mathrm{G}$ procedures, respectively (Table 2 ). Post-operative complications were similarly limited, with no ischaemic complications.

In addition to lower intraoperative complication rates, the favourable vision improvement and retinal attachment rates yielded a trend towards more positive outcomes with $23 \mathrm{G}$ compared to $20 \mathrm{G}$ vitrectomy. Surgery is more efficient and faster with $23 \mathrm{G}$ vitrectomy combined with more advanced fluidics, and this lends itself to less surgical trauma and lower risk surgery.

The main limitations of this study are due to its retrospective nature which takes into account the relative rarity of PSR cases, the surgeon-dependent approach/technique to surgery, case recruitment bias and uneven follow-up 

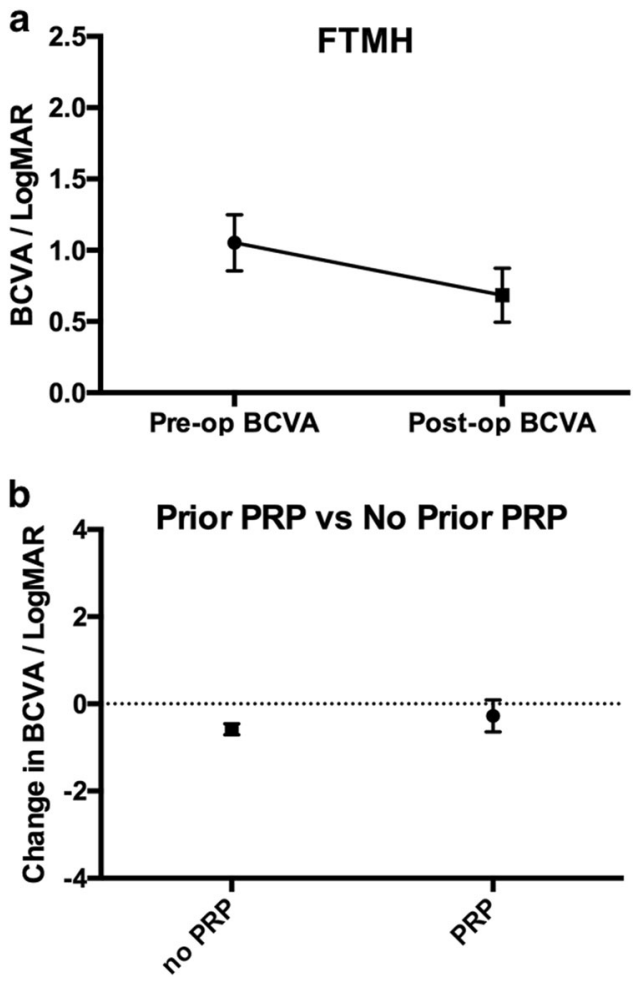

Fig. 3 a (top)-Mean improvement in mean BCVA after surgery for FTMH from 1.053 LogMAR to 0.685 LogMAR at final follow-up. b - Change in BCVA after surgery for patients with and without prior PRP laser

Table 2 - Per-operative complications of surgery for PSR

\begin{tabular}{lll}
\hline & $23 \mathrm{G}$ & $20 \mathrm{G}$ \\
\hline Post-operative & & \\
Fibrinous uveitis & 1 & 0 \\
Hypertensive uveitis & 0 & 1 \\
BRAO & 0 & 1 \\
Intra-operative & & \\
Entry site breaks & 3 & 3 \\
GRT & 0 & 2 \\
\hline
\end{tabular}

intervals. Future prospective comparisons could potentially involve a registry of such patients and surrogate measures such as duration of surgery and patient reported measures and outcomes for assessment of visual improvement.

The vitreoretinal service at our institution no longer advocates the routine use of PRP laser prophylaxis for PSR in view of the limited evidence in favour of PRP effects on PSR [9]. This was also demonstrated in our subgroup analysis of patients treated with pre-operative PRP vs. those without, where no significant difference in visual acuity outcomes was found (Fig. 3b). On an individual case-basis PRP is applied intraoperatively to treat capillary non-perfusion, with the use of short-pulse laser settings $(0.02 \mathrm{~s})$ recommended as these parameters cause less tissue damage and visual field loss [10, 11].

Our study highlights that advanced $23 \mathrm{G}$ vitrectomy and new surgical instrumentation and techniques have enabled favourable progression in clinical results for vitrectomy in PSR over the last decade. We report improved visual and anatomical outcomes with lower per-operative complication rates for patients with tractional and severe complications of sickle cell disease/ocular disease, but the differences were not statistically significant. In addition this is a retrospective analysis so concrete conclusions cannot be drawn from such results.

At our institution, we have 25 and $27 \mathrm{G}$ instrumentation available, and this may provide additional advantages for surgery and clinical outcomes in future, and this would be the basis of future work. Many of the sickle patients develop these vitreoretinal interface complications in their 30s and 40s, and the improved surgical outcomes will lead to improved quality of life in this cohort of young, workingage patients.

\section{Summary box}

\section{What was known before}

- Despite the high prevalence of autoinfarction, permanent visual loss occurs in over $10 \%$ of patients with proliferative sickle retinopathy.

- Only a few small studies have demonstrated improved outcomes from surgery for complications of PSR, and stabilisation of visual acuity remains guarded in tractional/rhegmatogenous detachments in these patients.

\section{What this study adds}

- $23 \mathrm{G}$ vitrectomy gives a better functional outcome with lower per-operative complication rates compared to $20 \mathrm{G}$ surgery in patients with complications of proliferative sickle retinopathy.

- Post-operative BCVA was maintained or improved in $76 \%$ of vitrectomised eyes that underwent vitrectomy for retinal detachment.

Acknowledgements We acknowledge expert advice and non-financial support from the NIHR London Biomedical Research Centre, the Vitreoretinal Service, and Dr Catherine Egan of the Medical Retina Service at Moorfields Eye Hospital.

\section{Compliance with ethical standards}

Conflict of interest The authors declare that they have no conflict of interest. 


\section{References}

1. Rees DC, Williams TN, Gladwin MT. Sickle-cell disease. Lancet. 2010;376:2018-31.

2. Elagouz M, Jyothi S, Gupta B, Sivaprasad S. Sickle cell disease and the eye: old and new concepts. Surv Ophthalmol. 2010;55:359-77.

3. Moriarty BJ, Acheson RW, Condon PI, Serjeant GR. Patterns of visual loss in untreated sickle cell retinopathy. Eye. 1988;2:330-5.

4. Condon PI, Serjeant GR. Behaviour of untreated proliferative sickle retinopathy. Br J Ophthalmol. 1980;64:404-11.

5. Williamson TH, Rajput R, Laidlaw DAH, Mokete B. Vitreoretinal management of the complications of sickle cell retinopathy by observation or pars plana vitrectomy. Eye. 2009;23:1314-20.

6. Chen RWS, Flynn HW, Lee WH, et al. Vitreoretinal management and surgical outcomes in proliferative sickle retinopathy: a case series. Am J Ophthalmol. 2014;157:870-5.e1.
7. Pulido JS, Flynn HW, Clarkson JG, Blankenship GW. Pars plana vitrectomy in the management of complications of proliferative sickle retinopathy. Arch Ophthalmol. 1988;106:1553-7.

8. Jampol LM, Green JL, Goldberg MF, Peyman GA. An update on vitrectomy surgery and retinal detachment repair in sickle cell disease. Arch Ophthalmol. 1982;100:591-3.

9. Myint KT, Sahoo S, Thein AW, Moe S, Ni H. Laser therapy for retinopathy in sickle cell disease. Cochrane Database Syst Rev. 2015;CD010790.

10. Muqit MM, Denniss J, Nourrit V, et al. Spatial and spectral imaging of retinal laser photocoagulation burns. Invest Ophthalmol Vis Sci. 2011:52:994-1002.

11. Wang Y, Muqit MM, Stanga PE, Young LB, Henson DB. Spatial changes of central field loss in diabetic retinopathy after laser. Optom Vis Sci. 2014;91:111-20. 